Research Article

\title{
Bioinformatics Analysis of the Molecular Mechanism and Potential Treatment Target of Ankylosing Spondylitis
}

\author{
Fanyan Meng, ${ }^{1}$ Ningna Du, ${ }^{1}$ Daoming Xu, ${ }^{1}$ Li Kuai, ${ }^{1}$ Lanying Liu, ${ }^{1}$ and Minning Xiu $\mathbb{D}^{2}$ \\ ${ }^{1}$ Department of Acupuncture and Rehabilitation, Jiangsu Provincial Hospital of Traditional Chinese Medicine, China \\ ${ }^{2}$ Department of Nursing, Jiangsu Provincial Hospital of Traditional Chinese Medicine, 155 Hanzhong Road, Nanjing, China \\ Correspondence should be addressed to Minning Xiu; xmn1972@126.com
}

Received 17 April 2021; Accepted 5 June 2021; Published 22 July 2021

Academic Editor: Tao Huang

Copyright (C) 2021 Fanyan Meng et al. This is an open access article distributed under the Creative Commons Attribution License, which permits unrestricted use, distribution, and reproduction in any medium, provided the original work is properly cited.

\begin{abstract}
Ankylosing spondylitis (AS) is an autoimmune disease that mainly affects the spinal joints, sacroiliac joints, and adjacent soft tissues. We conducted bioinformatics analysis to explore the molecular mechanism related to AS pathogenesis and uncover novel potential molecular targets for the treatment of AS. The profiles of GSE25101, containing gene expression data extracted from the blood of 16 AS patients and 16 matched controls, were acquired from the Gene Expression Omnibus (GEO) database. The background correction and standardization were carried out utilizing the transcript per million (TPM) method. After analysis of AS patients and the normal groups, we identified 199 differentially expressed genes (DEGs) with upregulation and 121 DEGs with downregulation by the limma R package. The results of the Kyoto Encyclopedia of Genes and Genomes (KEGG) pathway and Gene Ontology (GO) biological process enrichment analysis revealed that the DEGs with upregulation were mainly associated with spliceosome, ribosome, RNA-catabolic process, electron transport chain, etc. And the DEGs with downregulation primarily participated in $\mathrm{T}$ cell-associated pathways and processes. After analysis of the protein-protein interaction (PPI) network, our data revealed that the hub genes, comprising MRPL13, MRPL22, LSM3, COX7A2, COX7C, EP300, PTPRC, and CD4, could be the treatment targets in AS. Our data furnish new hints to uncover the features of AS and explore more promising treatment targets towards AS.
\end{abstract}

\section{Background}

Ankylosing spondylitis (AS), which mostly occurs in the sacroiliac joints, spine, and external joints (1), is a rheumatic immune disease with an incidence of $0.3 \%$ in China (2). AS patients are mostly young men (3). Most patients have early symptoms like dull pain in the waist, buttocks, and sacroiliac areas, later with complications in the heart, eyes, ears, and nervous system (4). The specific pathogenic mechanism of AS is not yet clear. It is only understood that genetic factors play major roles in AS pathogenesis (5). Besides, environmental, immune, metabolic, and other factors are also common causes of AS (6). Presently, AS cannot be completely cured. Most patients can be treated with nonsteroidal drugs and exercise. In severe cases, surgery is required (7). It should be noted that AS does not affect the survival of patients, but it will gradually ruin their lives (4). Therefore, to avoid the aggravation of AS patients, new treatment methods need to be developed as soon as possible.

Bioinformatics is a new interdisciplinary subject that takes computer as a tool to store, retrieve, and analyze biological information in the study of life sciences (8). At present, the research focus of bioinformatics is mainly embodied in genomics and proteomics (9), which is to analyze the biological information of the structure and function expressed in the sequence from the point of view of nucleic acid and protein sequences. Bioinformatics also plays an important role in the study of human diseases (10). Such as the establishment of disease-related bioinformatics database (11), isolation and identification of human genes and disease-related genes (12), and accelerating the development of gene drugs (13), these have an important and positive impact on the prevention and treatment of human diseases. We here executed bioinformatics analysis to investigate the molecular mechanism 


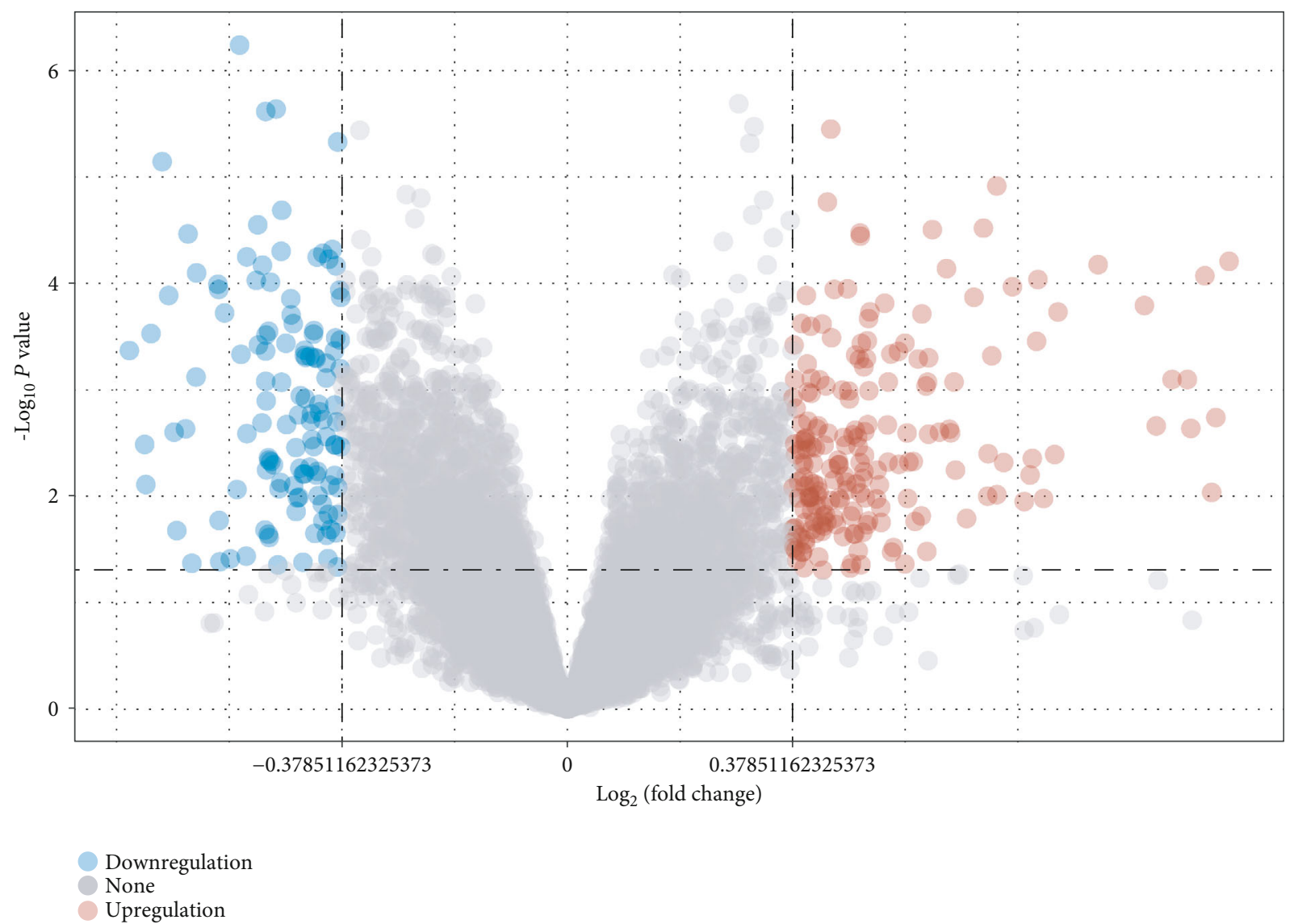

(a)

Figure 1: Continued. 


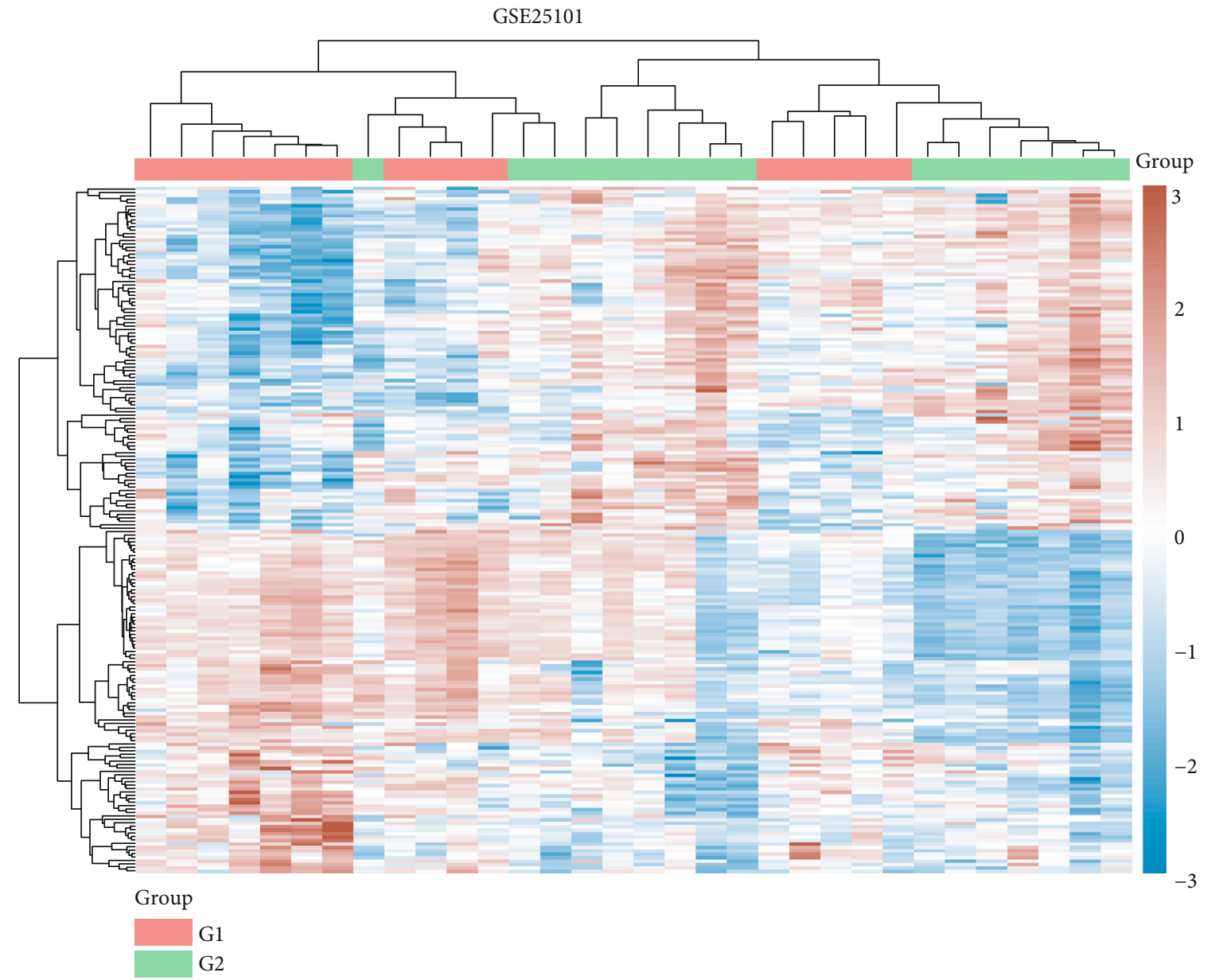

(b)

FIgURE 1: (a) Volcano plot and (b) heat map show the DEGs between AS patients and normal controls. Blue indicates low expression values, and red represents high expression values. G1 and G2 represent AS patients and the normal control group, respectively.

of AS pathogenesis and dig out the unclear details and probable treatments towards AS. GSE25101 was utilized to detect the differentially expressed genes (DEGs) in the specimens of AS patients and normal controls. Then, the molecular mechanism of AS was obtained after analyzing the pathway and functional enrichment. Finally, with the use of these DEGs, we established the protein-protein interaction (PPI) network to seek prospective genes in targeting AS.

\section{Material and Methods}

2.1. Data Source. GSE25101 (14), as the RNA expression profiles, was downloaded from the Gene Expression Omnibus database (GEO, https://www.ncbi.nlm.nih.gov/geo/) in NCBI. GSE25101 contained RNA expression data extracted from the blood of 16 AS patients and 16 matched controls using PAXgene tubes (15).

2.2. Data Preprocessing and the DEG Screening. The expression data of the GSE25101 data set was normalized by the transcript per million (TPM) method. Ensembl transcript IDs were transformed into the symbols of genes. The mean value was regarded as the expression level of genes if diverse probes were annotated to the same genes. Then, we utilized the limma R package to screen the DEGs between AS patients and normal control groups. The cut-off setting was $P<0.05$ and $\mid \log _{2}$ fold change $\mid>0.378511$ based on the Benjamini and Hochberg $(\mathrm{BH})$ procedure. Morpheus was applied to draw the heat map based on the website (https://software .broadinstitute.org/morpheus/).

2.3. Analysis of Pathway and Functional Enrichment. Metascape was applied to analyze the Kyoto Encyclopedia of Genes and Genomes (KEGG) pathway and Gene Ontology (GO) biological process enrichment (16) by accessing at https:// metascape.org/gp/index.html\#/main/step1. All genomic genes are thought of as enriched background. We obtained the top 20 significantly enriched terms with regard to the $P$ values which were obtained on the basis of the accumulative hypergeometric distribution.

2.4. Analysis of the PPI Network. The Search Tool for the Retrieval of Interacting Genes (17) (STRING, https://stringdb.org) was executed to analyze and visualize the PPI network of upregulated and downregulated DEGs. A medium confidencescore $>0.400$ indicated there was a great 


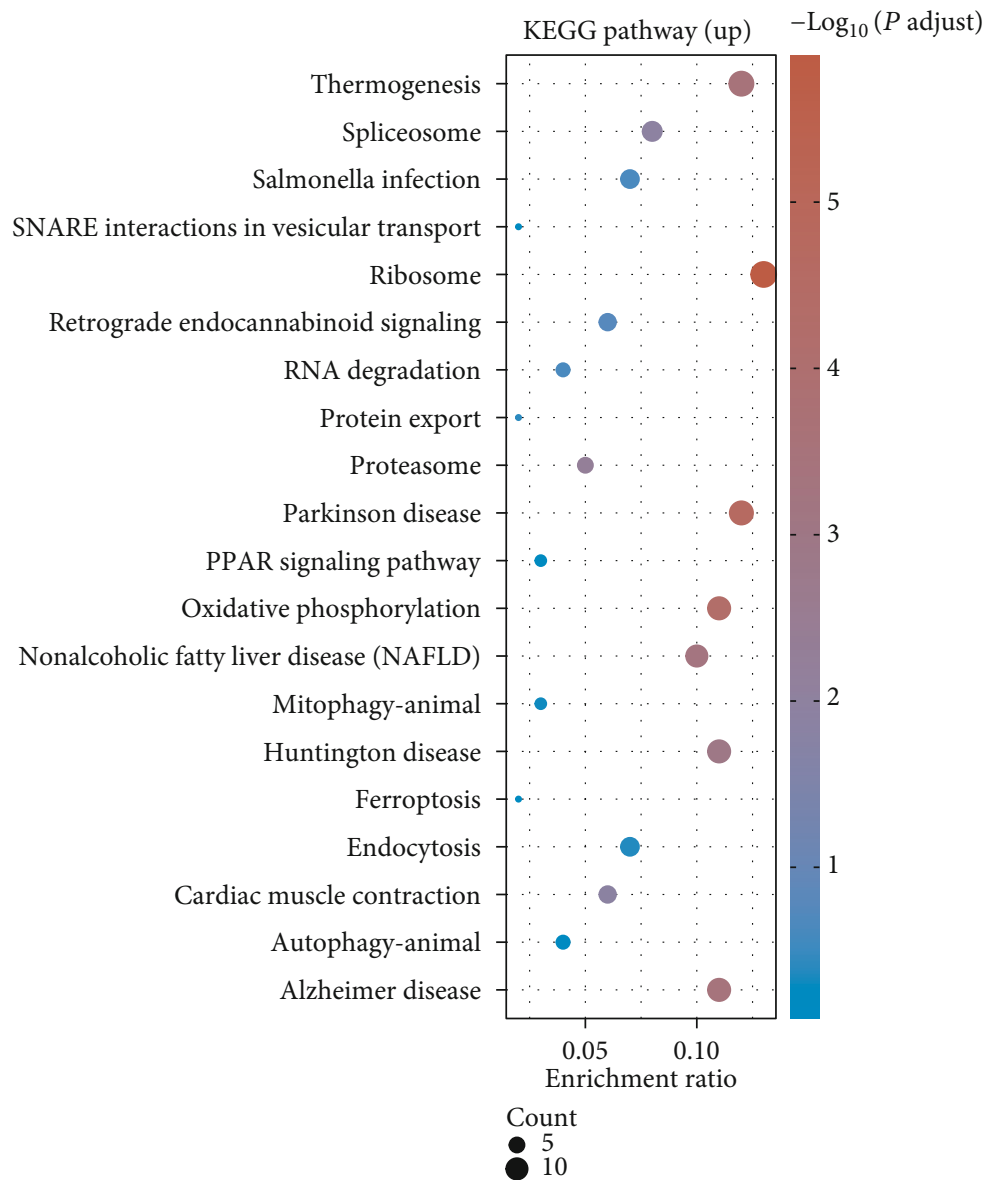

(a)

Figure 2: Continued. 


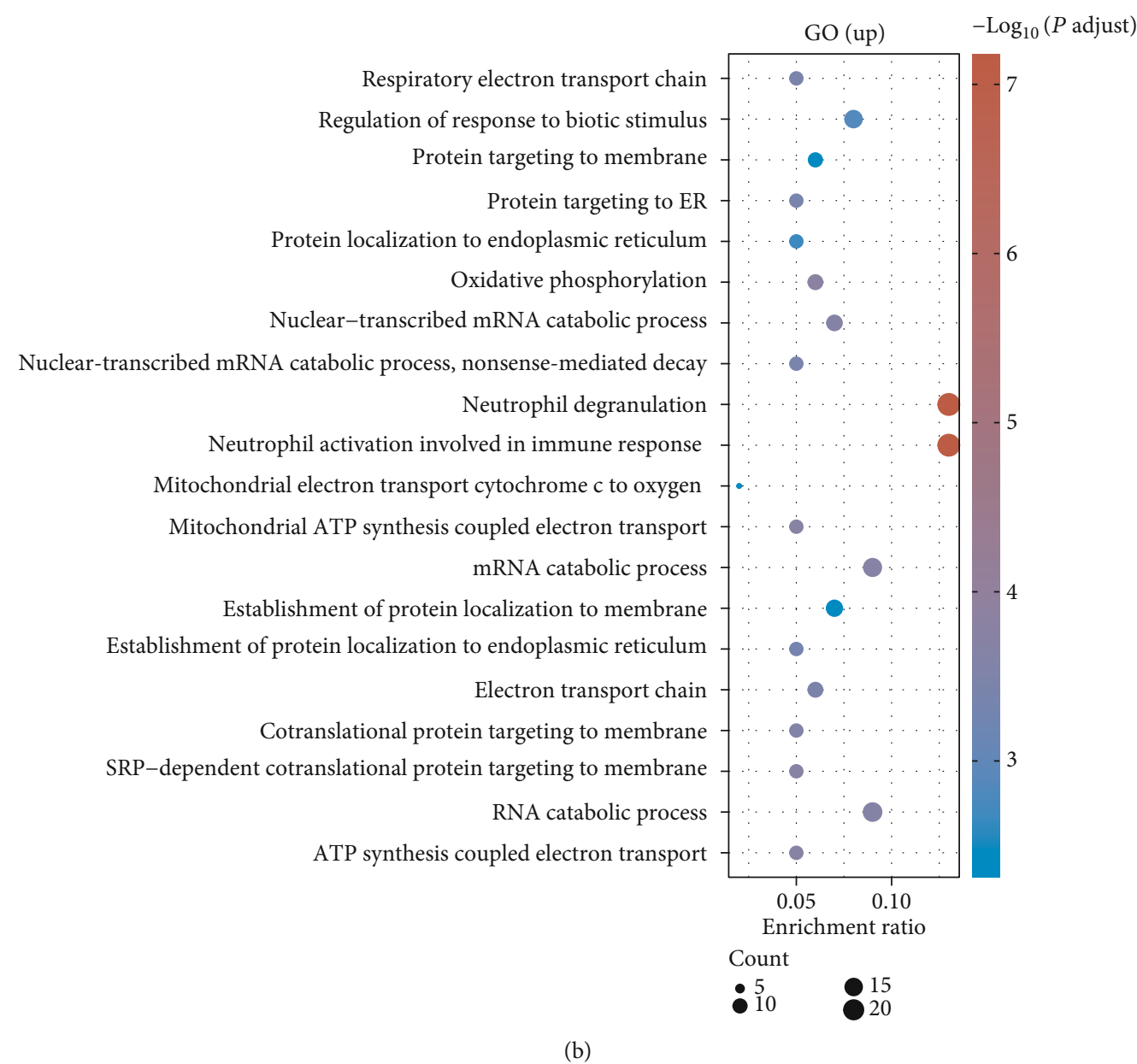

FIGURE 2: (a) KEGG pathway and (b) GO biological process enrichment analysis of upregulated DEGs using Metascape. The top 20 significantly enriched terms are presented.

difference. Moreover, hub genes, as pivotal candidate genes with essential physiological regulatory use, were selected based on their degree of importance.

\section{Results}

3.1. Data Preprocessing and the DEG Screening. TPM was exploited to standardize the transcriptome expression data of the GSE126118 dataset. The limma R package was taken to screen the DEGs between AS patients and matched control groups. The volcano plot showed the identified DEGs (Figure 1(a)). According to the critical criteria of $P$ value $<$ 0.05 and $\mid \log _{2}$ (fold change) $\mid>0.378511,320$ DEGs were screened. Among these, 199 DEGs were increased and 121 DEGs were decreased. In addition, we also constructed the gene expression heat map with color patterns to indicate the difference of gene expression existing in AS patients and normal groups (Figure 1(b)).

3.2. Analysis of Pathway and Functional Enrichment. Given the ontology sources, including KEGG pathways and GO biological processes, we analyzed pathways and functional enrichment of the DEGs by Metascape. All genes were regarded as the enrichment background. According to the calculated $P$ values in the light of accumulative hypergeometric distribution, the top 20 with statistically obvious KEGG pathways and GO biological processes associated with upregulated and downregulated DEGs were presented in Figures 2 and 3, respectively. It could be observed that the DEGs with upregulation primarily participated in RNA- and protein-associated pathways, such as spliceosome, ribosome, oxidative phosphorylation, proteasome, RNA degradation, and protein export (Figure 2(a)). The enriched biological process of upregulated DEGs was related to energy production such as respiratory electron transport chain, oxidative phosphorylation, cytochrome $\mathrm{c}$ to oxygen mitochondrial ATP synthesis coupled electron transport, and ATP synthesis coupled electron transport (Figure 2(b)). The downregulated DEGs were enriched in pathways and processes associated with the immune response, especially Th17, Th1, and Th2 cell differentiation; T cell receptor signaling pathway; antigen processing and presentation; positive $\mathrm{T}$ cell selection; lymphocyte differentiation; lymphocyte activation touching upon the immune response; immune response-activating cell surface receptor signaling pathway; antigen receptor-mediated signaling pathway; $\mathrm{T}$ cell selection; and activation and differentiation (Figures $3(\mathrm{a})$ and $3(\mathrm{~b})$ ). 


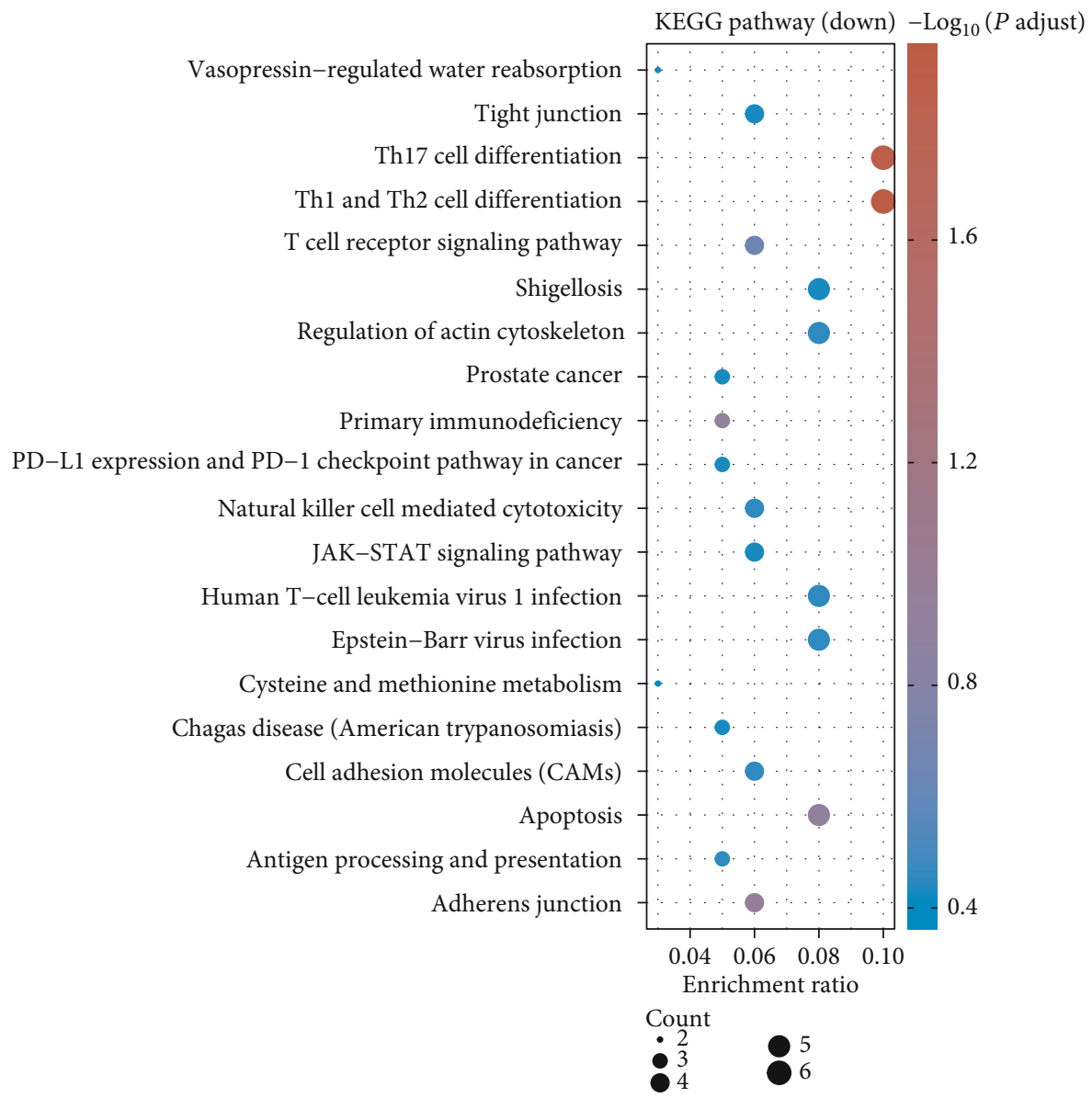

(a)

Figure 3: Continued. 


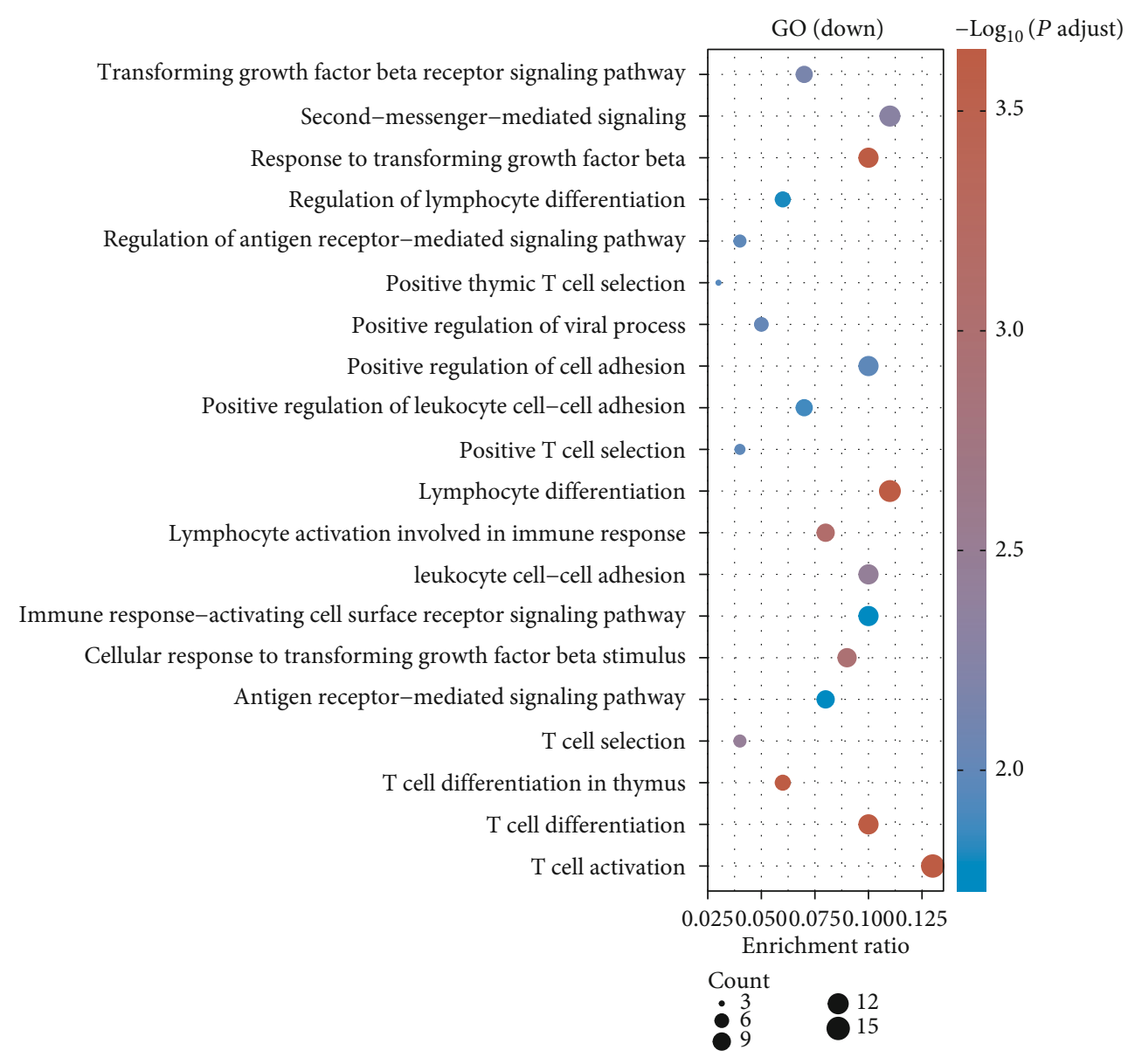

(b)

Figure 3: (a) KEGG pathway and (b) GO biological process enrichment analysis of downregulated DEGs using Metascape. The top 20 significantly enriched terms are presented.

3.3. PPI Network Analysis. The STRING software for constructing the PPI network was utilized to investigate the interplay among the DEGs. Data from the STRING database revealed that these DEGs interacted with each other. Figure 4 showed that the PPI network of upregulated DEGs was composed of 198 nodes and 734 edges. Among the 198 nodes, MRPL13 (degree $=26), \quad$ LSM3 (degree $=25), \quad$ COX7A2 $($ degree $=24), \quad$ COX7C $\quad($ degree $=24), \quad$ and MRPL22 (degree $=23)$ were the top 5 significant hub node genes. Also, the PPI network of downregulated DEGs (Figure 5) contained 120 nodes and 159 edges. Among the 120 nodes, EP300 (degree $=15), \quad$ PTPRC $($ degree $=15)$, and CD4 (degree $=14)$ were the top 3 significant hub node genes.

\section{Discussion}

AS is a complex disease that involves many factors (6). For patients with AS, there is currently no complete cure. The only way for patients to improve the poor prognosis is to actively cooperate with treatment and perform some rehabilitation exercises. Besides, early detection and early treatment are very necessary for patients. There is evidence showing that some lncRNAs are also involved in the development of
AS. Some research shows that HLA-B27 may be involved in the pathogenesis (18). For example, $\mathrm{Li}$ et al. reported that the expression of IncRNA MEG 3 was downregulated in AS (19), which affected the length of the patient's hospital stay. Tan et al. proposed that the pathogenesis of AS might be related to insufficient autophagy and downregulation of lncRNA GAS 5, and lncRNA GAS 5 might have clinical application value (20). Our discovery gives a new hint of uncovering the traits of AS and exploiting a prospective target for AS treatment.

Our study attempted to explore the underlying mechanisms related to the AS. Public gene expression data from the GEO database GSE25101 were applied to analyze the data. To be first, we normalized the raw data and dug out the DEGs existing in AS patients and normal controls. Then, we performed the KEGG and GO analyses to detect these DEG-related pathways and biological processes. We identified 199 DEGs with increase and 121 DEGs with a reduction from a PPI network. The data of enrichment analysis revealed that the DEGs with upregulation majorly participated in the spliceosome, ribosome, RNA-catabolic process, electron transport chain, and so on. The DEGs with downregulation mostly took part in $\mathrm{T}$ cell- 


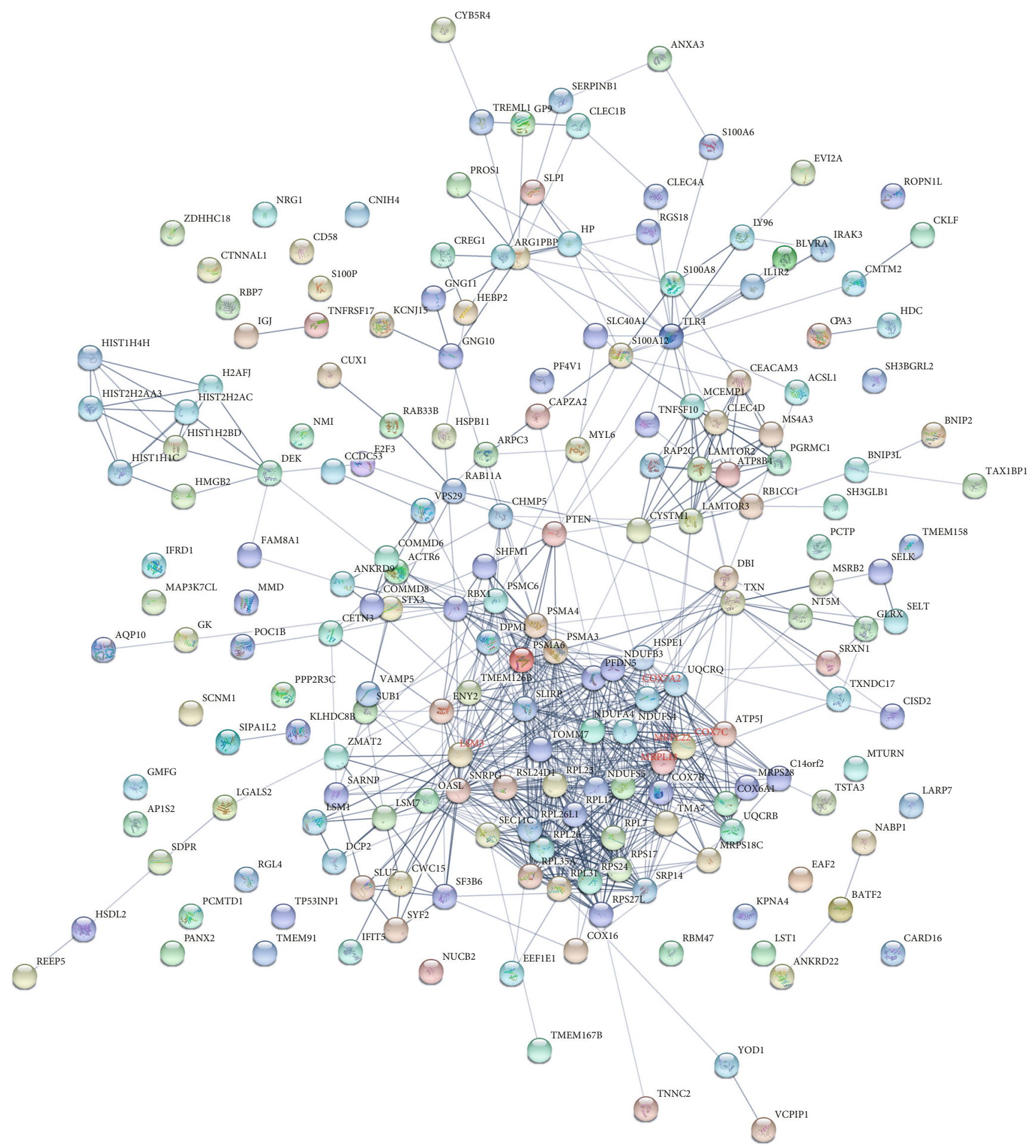

FIgURE 4: Analysis of the PPI network of the DEGs with upregulation by the STRING database. Circle and line represent the protein and interaction, respectively.

associated pathways and processes. Studies have shown that some new congenital or physical diseases are related to mutations in secondary spliceosome components (21), such as cerebellar ataxia, myelodysplastic syndrome, spondyloepiphyseal dysplasia tarda, amyotrophic lateral sclerosis, and spinal muscular atrophy. The generation of reactive oxygen species (ROS) by the mitochondrial elec- tron transport chain (ETC) is thought to be important in the pathogenesis of neurodegenerative diseases such as the aging process and Parkinson's disease (22). The PPI network analysis revealed that MRPL13, MRPL22, LSM3, COX7A2, and COX7C were the hub upregulated genes in AS, and EP300, PTPRC, and CD4 were the hub genes with downregulation in AS. 


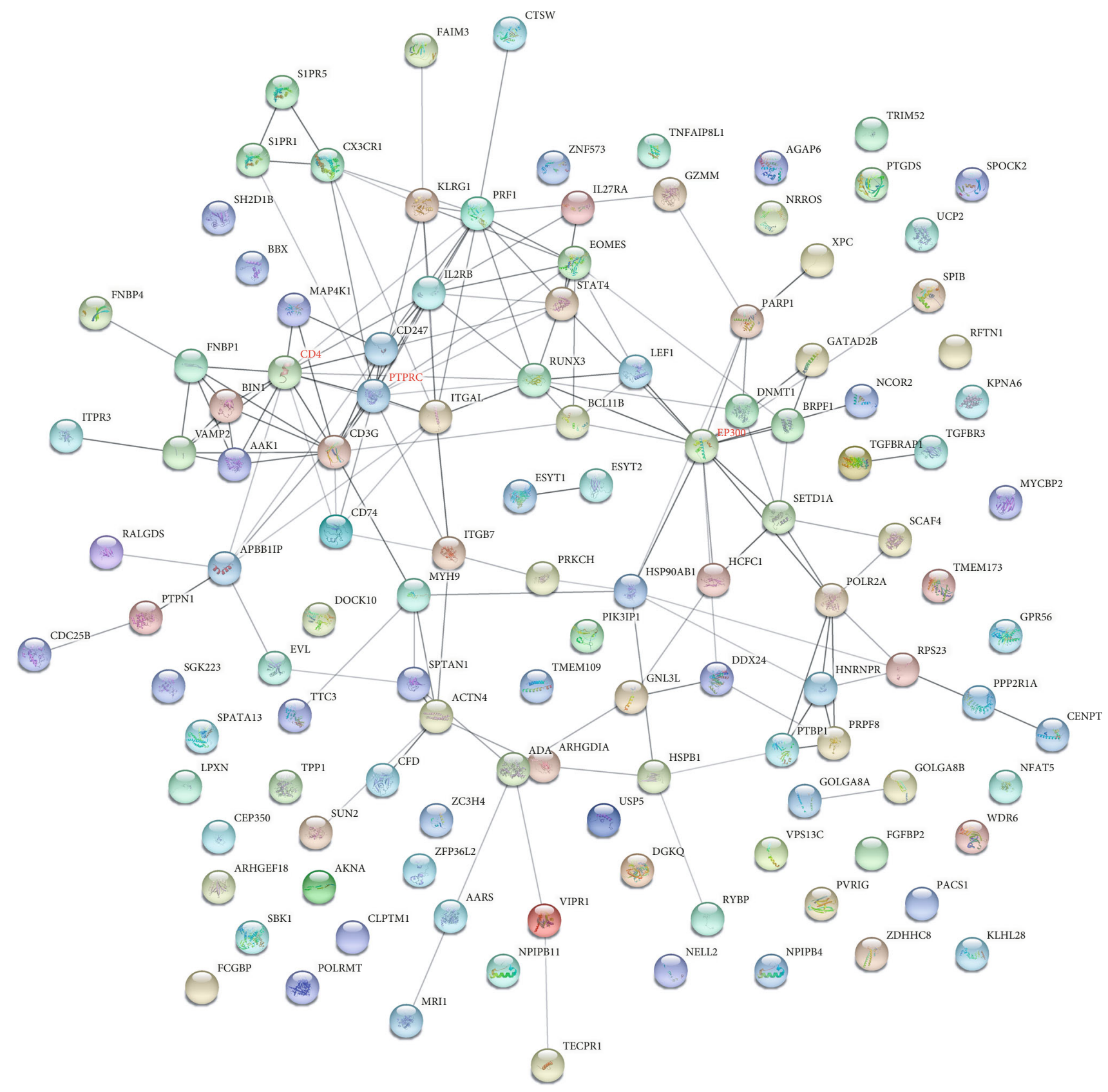

FIgURE 5: Analysis of the PPI network of the DEGs with downregulation by the STRING database. Circle and line represent the protein and interaction, respectively.

Nuclear genes encode MRPL13 and MRPL22 which are the members of mitochondrial ribosomal proteins (MRPs). MRPL13 and MRPL22 are generated in the cytoplasm and then transported to mitochondria to assemble mitochondrial ribosomes (23). MRPs are essential for mitochondrial oxidative phosphorylation and exhibit crucially in regulating apoptosis-inducing factors (24). Aberrant MRP expressions will result in multiple dysregulations, such as disordered mitochondrial metabolism and dysfunctional cell (25). Here, we report abnormally expressed MRPs in AS.

LSM3, as a constituent of the precatalytic spliceosome, participates in the assembly of the spliceosome (26). It functions essentially in the process of pre-mRNA splicing as a part of the U4/U6-U5 tri-snRNP (27). Pre-mRNA splicing displays an essential role in the generation of mature mRNAs in eukaryotic cells to regulate gene expression (28). Combined with the enrichment analysis, our results suggested that the AS exhibited a relation with the spliceosome. COX7A2 and COX7C are the components of the cytochrome $c$ oxidase (COX) which is the terminal enzyme of the mitochondrial respiratory chain. COX is responsible for catalyzing the reduction of oxygen to water, which is critical for energy production in all organisms (29). The COX-related pathway contains ATP synthesis, respiratory electron transport, heat production, and so on, consistent with our enrichment analysis. 
Ep300 encodes a p300 transcriptional coactivator associated with adenovirus E1A (30). Histone acetyltransferase Ep300 mediates the modulation of transcription via remodeling chromatin, cell proliferation, and differentiation (31). Ep300 has also been reported as a coactivator of HIF1A (hypoxia-inducible factor 1 alpha) thus activating VEGF which is a hypoxia-induced gene (32). Diseases associated with EP300 contain Menke-Hennekam Syndrome 2 and RubinsteinTaybi Syndrome 2 (33). Pathways related to EP300 contain regulating TP53 activity through acetylation and Nur77 signaling in the $\mathrm{T}$ cell. Protein Tyrosine Phosphatase Receptor Type C (PTPRC) belongs to the tyrosine phosphatase family, generally functioning as a regulator of several cellular processes, i.e., mitosis, cell proliferation and differentiation, and oncogenic transformation (34). PTPRC is shown to be a fundamental molecule in regulating $\mathrm{T}$ cell and $\mathrm{B}$ cell antigen receptor signaling (35). PTPRC-related signaling works via direct interaction with the antigen receptor complex or through motivating several kinases of the Src family. CD4 membrane glycoprotein, also named as the CD4 antigen, is encoded by the CD4 gene of T lymphocytes (36). Not only do T lymphocytes express CD4 genes, but also macrophages, $\mathrm{B}$ cells, granulocytes, and various regions of the brain do. The CD4 membrane glycoprotein along with $\mathrm{T}$ cell receptors works together as receptors to recognize antigens presented by antigen-presenting cells under the circumstance of class II MHC molecules (37). It can activate or enhance the early stage of $\mathrm{T}$ cell activation and exhibit as a central mediator of indirect neuronal damage in infectious and immunemediated diseases of the central nervous system. To sum up, these three genes are associated with $\mathrm{T}$ cells which are consistent with our enrichment analysis of downregulated genes and could be the treatment targets.

The study has some limitations. The expression level of the hub gene in AS needs further verification. In the following study, we will collect clinical samples and determine the expression level of the hub gene through TaqMan RealTime PCR. We will further explore the correlation between hub gene expression and clinical parameters (including clinical stage, age, and survival time).

\section{Conclusion}

This study used the bioinformatics method to analyze gene expression data of GSE25101 to unearth the considerable points and promising targets in AS treatment. In total, we screened 199 DEGs with upregulation and 121 DEGs with downregulation after analyzing AS patients and normal control ones. KEGG and GO enrichment analyses demonstrated that the upregulated DEGs were largely associated with spliceosome, ribosome, RNA-catabolic process, and electron transport chain. And the DEGs with downregulation were generally enriched in $\mathrm{T}$ cell-associated pathways and processes. PPI network analysis revealed that MRPL13, MRPL22, LSM3, COX7A2, COX7C, EP300, PTPRC, and CD4 were the hub genes in AS and could be the treatment targets. Our data furnish new hints to uncover the features of AS and explore more promising treatment targets towards
AS. In the medical field, it has positive and important significance for the future treatment of AS.

\section{Data Availability}

The datasets used and/or analyzed during the current study are available from the corresponding author on reasonable request.

\section{Conflicts of Interest}

The authors declare no competing financial interests.

\section{Authors' Contributions}

Conception and design were handled by Fanyan Meng and Lanying Liu. Development of methodology was handled by Minning Xiu and Ningna Du. Sample collection was handled by Ningna $\mathrm{Du}$, Daoming Xu, and Li Kuai. Analysis and interpretation of data were handled by Fanyan Meng and Minning Xiu. Writing, review, and/or revision of the manuscript were handled by Fanyan Meng, Ningna Du, and Lanying Liu. Fanyan Meng and Ningna Du contributed equally to this work.

\section{Acknowledgments}

This work is supported by the Natural Science Foundation of the Hospital-Level Project of the Jiangsu Provincial Hospital of Traditional Chinese Medicine (Y20056, applicant: Fanyan Meng).

\section{References}

[1] I. Sudol-Szopinska and A. Urbanik, "Diagnostic imaging of sacroiliac joints and the spine in the course of spondyloarthropathies," Polish Journal of Radiology, vol. 78, no. 2, pp. 43-49, 2013.

[2] S. I. Davidson, X. Wu, Y. Liu et al., "Association of ERAP1, but not IL23R, with ankylosing spondylitis in a Han Chinese population," Arthritis and Rheumatism, vol. 60, no. 11, pp. 32633268, 2009.

[3] E. N. Kubiak, R. Moskovich, T. J. Errico, and P. E. Di Cesare, "Orthopaedic management of ankylosing spondylitis," The Journal of the American Academy of Orthopaedic Surgeons, vol. 13, no. 4, pp. 267-278, 2005.

[4] J. Wolf and P. Fasching, "Ankylosing spondylitis," Wiener Medizinische Wochenschrift (1946), vol. 160, no. 9-10, pp. 211-214, 2010.

[5] M. Mahmoudi, S. Aslani, M. H. Nicknam, J. Karami, and A. R. Jamshidi, "New insights toward the pathogenesis of ankylosing spondylitis; genetic variations and epigenetic modifications," Modern Rheumatology, vol. 27, no. 2, pp. 198-209, 2017.

[6] A. Voruganti and P. Bowness, "New developments in our understanding of ankylosing spondylitis pathogenesis," Immunology, vol. 161, no. 2, pp. 94-102, 2020.

[7] M. Dougados, B. Dijkmans, M. Khan, W. Maksymowych, S. van der Linden, and J. Brandt, "Conventional treatments for ankylosing spondylitis," Annals of the Rheumatic Diseases, vol. 61, Supplement 3, pp. 40iii-40i50, 2002. 
[8] J. Dopazo, "Bioinformatics and cancer: an essential alliance," Clinical \& Translational Oncology, vol. 8, no. 6, pp. 409-415, 2006.

[9] C. Kumar and M. Mann, "Bioinformatics analysis of mass spectrometry-based proteomics data sets," FEBS Letters, vol. 583, no. 11, pp. 1703-1712, 2009.

[10] X. Wang and L. Liotta, "Clinical bioinformatics: a new emerging science," Journal of Clinical Bioinformatics, vol. 1, no. 1, p. 1, 2011.

[11] A. R. Wattam, D. Abraham, O. Dalay et al., "PATRIC, the bacterial bioinformatics database and analysis resource," Nucleic Acids Research, vol. 42, no. D1, pp. D581-D591, 2014.

[12] N. Yuyama, D. E. Davies, M. Akaiwa et al., "Analysis of novel disease-related genes in bronchial asthma," Cytokine, vol. 19, no. 6, pp. 287-296, 2002.

[13] Y. Li, D. M. Umbach, J. M. Krahn, I. Shats, X. Li, and L. Li, "Predicting tumor response to drugs based on geneexpression biomarkers of sensitivity learned from cancer cell lines," BMC Genomics, vol. 22, no. 1, p. 272, 2021.

[14] K. Chen, Y. Zhao, Y. Chen et al., "A sub-pathway based method to identify candidate agents for ankylosing spondylitis," Molecules, vol. 17, no. 10, pp. 12460-12468, 2012.

[15] M. Kruhøffer, L. Dyrskjøt, T. Voss et al., "Isolation of microarray-grade total RNA, microRNA, and DNA from a single PAXgene blood RNA tube," The Journal of Molecular Diagnostics, vol. 9, no. 4, pp. 452-458, 2007.

[16] F. Yuan, L. Lu, Y. Zhang, S. Wang, and Y. D. Cai, "Data mining of the cancer-related lncRNAs GO terms and KEGG pathways by using mRMR method," Mathematical Biosciences, vol. 304, pp. 1-8, 2018.

[17] D. Szklarczyk, A. L. Gable, D. Lyon et al., "STRING v11: protein-protein association networks with increased coverage, supporting functional discovery in genome-wide experimental datasets," Nucleic Acids Research, vol. 47, no. D1, pp. D607D613, 2019.

[18] R. A. Colbert, T. M. Tran, and G. Layh-Schmitt, "HLA-B27 misfolding and ankylosing spondylitis," Molecular Immunology, vol. 57, no. 1, pp. 44-51, 2014.

[19] Y. Li, S. Zhang, C. Zhang, and M. Wang, "IncRNA MEG3 inhibits the inflammatory response of ankylosing spondylitis by targeting miR-146a," Molecular and Cellular Biochemistry, vol. 466, no. 1-2, pp. 17-24, 2020.

[20] M. Tan, Q. B. Zhang, T. H. Liu et al., “Autophagy dysfunction may be involved in the pathogenesis of ankylosing spondylitis," Experimental and Therapeutic Medicine, vol. 20, no. 4, pp. 3578-3586, 2020.

[21] B. Verma, M. V. Akinyi, A. J. Norppa, and M. J. Frilander, "Minor spliceosome and disease," Seminars in Cell \& Developmental Biology, vol. 79, pp. 103-112, 2018.

[22] Y. Liu, G. Fiskum, and D. Schubert, "Generation of reactive oxygen species by the mitochondrial electron transport chain," Journal of Neurochemistry, vol. 80, no. 5, pp. 780-787, 2002.

[23] J. Kaur and R. A. Stuart, "Truncation of the Mrp20 protein reveals new ribosome-assembly subcomplex in mitochondria," EMBO Reports, vol. 12, no. 9, pp. 950-955, 2011.

[24] A. J. Slot, S. V. Molinski, and S. P. Cole, "Mammalian multidrug-resistance proteins (MRPs)," Essays in Biochemistry, vol. 50, no. 1, pp. 179-207, 2011.

[25] G. Gopisetty and R. Thangarajan, "Mammalian mitochondrial ribosomal small subunit (MRPS) genes: a putative role in human disease," Gene, vol. 589, no. 1, pp. 27-35, 2016.
[26] C. L. Will and R. Luhrmann, "Spliceosome structure and function," Cold Spring Harbor Perspectives in Biology, vol. 3, no. 7, 2011.

[27] L. Zhou, J. Hang, Y. Zhou et al., "Crystal structures of the Lsm complex bound to the 3' end sequence of U6 small nuclear RNA," Nature, vol. 506, no. 7486, pp. 116-120, 2014.

[28] T. Maniatis and B. Tasic, "Alternative pre-mRNA splicing and proteome expansion in metazoans," Nature, vol. 418, no. 6894, pp. 236-243, 2002.

[29] A. Timon-Gomez, E. Nyvltova, L. A. Abriata, A. J. Vila, J. Hosler, and A. Barrientos, "Mitochondrial cytochrome $c$ oxidase biogenesis: recent developments," Seminars in Cell \& Developmental Biology, vol. 76, pp. 163-178, 2018.

[30] R. H. Goodman and S. Smolik, "CBP/p300 in cell growth, transformation, and development," Genes \& Development, vol. 14, no. 13, pp. 1553-1577, 2000.

[31] H. Lehrmann, L. L. Pritchard, and A. Harel-Bellan, "Histone acetyltransferases and deacetylases in the control of cell proliferation and differentiation," Advances in Cancer Research, vol. 86, pp. 41-65, 2002.

[32] H. Palma-Gudiel, E. Eixarch, F. Crispi, S. Moran, A. S. Zannas, and L. Fananas, "Prenatal adverse environment is associated with epigenetic age deceleration at birth and hypomethylation at the hypoxia-responsive EP300 gene," Clinical Epigenetics, vol. 11, no. 1, p. 73, 2019.

[33] Q. Wang, W. Xu, Y. Liu, and H. Yuan, “A novel CREBBP inframe deletion variant in a Chinese girl with atypical Rubinstein-Taybi syndrome phenotypes," Journal of Molecular Neuroscience, vol. 71, no. 3, pp. 607-612, 2021.

[34] T. Yi and D. Lindner, "The role and target potential of protein tyrosine phosphatases in cancer," Current Oncology Reports, vol. 10, no. 2, pp. 114-121, 2008.

[35] J. G. Cyster and C. C. Goodnow, "Protein tyrosine phosphatase $1 \mathrm{C}$ negatively regulates antigen receptor signaling in B lymphocytes and determines thresholds for negative selection," Immunity, vol. 2, no. 1, pp. 13-24, 1995.

[36] D. R. Littman, "The structure of the CD4 and CD8 genes," Annual Review of Immunology, vol. 5, no. 1, pp. 561-584, 1987.

[37] A. Veillette, M. A. Bookman, E. M. Horak, and J. B. Bolen, "The CD4 and CD8 T cell surface antigens are associated with the internal membrane tyrosine-protein kinase p56 ${ }^{\text {lck }}$, Cell, vol. 55, no. 2, pp. 301-308, 1988. 